\title{
ON A STABILITY PROPERTY OF NONLINEAR SYSTEMS WITH PERIODIC INPUTS HAVING SLOWLY VARYING AVERAGE
}

\author{
Y. U. Choi, H. Shim, and J.H. Seo
}

\author{
ASRI, School of Electrical Engineering, \\ Seoul National University, Kwanak P.O.Box 34, \\ Seoul, 151-600, Korea. Email: hshim@snu.ac.kr
}

\begin{abstract}
It is known that, if an equilibrium of a nonlinear system has a stability property when an external input is frozen, then the property is maintained under the input being slowly varying. In this paper, we show that the same stability property is preserved not only under slowly varying input but also under slowlyvarying-average input (which is not actually slowly varying but its 'average' is slowly varying). The input is assumed to be periodic and to vary sufficiently fast. We prove the claim by the average theory and some previous results on the slowly varying inputs. Copyright (c) 2005 IFAC
\end{abstract}

Keywords: Average theory; Slowly varying input; Stability of nonlinear systems

\section{INTRODUCTION}

In the early 1990s, (Kelemen, 1986), (Lawrence and Rugh, 1990), and (Khalil and Kokotović, 1991) have presented a stability property of nonlinear systems with slowly varying inputs, which can now be found in a graduate textbook such as (Khalil, 1996). The goal of this paper is to extend the result of (Kelemen, 1986; Lawrence and Rugh, 1990; Khalil and Kokotović, 1991) in the sense that the same stability property is established under periodic inputs having slowly varying 'average.' In other words, we consider an input that may not be slowly varying itself, but whose average is slowly varying.

To be concrete, let us consider a dynamical system given by

$$
\dot{x}=f(x, u),
$$

where $f(\cdot, \cdot)$ is continuously differentiable, $x \in$ $\mathbb{R}^{n}, u \in \Gamma \subset \mathbb{R}^{m}$ in which $\Gamma$ is a connected compact set. Suppose that, for each frozen (i.e., constant) input $u \in \Gamma$, there exists a corresponding equilibrium $x^{*}(u)$ such that $f\left(x^{*}(u), u\right)=0$.
Suppose also that, for any two (constant) inputs $u_{1}$ and $u_{2}$ contained in $\Gamma$, we want to drive the state $x(t)$, which is initially located in a neighborhood of $x^{*}\left(u_{1}\right)$, into a neighborhood of $x^{*}\left(u_{2}\right)$. This problem has been dealt with in (Kelemen, 1986; Lawrence and Rugh, 1990; Khalil and Kokotović, 1991), where it is proved that, if there exists a curve $\mathcal{U}$ in $\Gamma$ that connects two points $u_{1}$ and $u_{2}$ and, for each fixed $\bar{u}$ on the curve $\mathcal{U}$, the corresponding equilibrium $x^{*}(\bar{u})$ is locally exponentially ${ }^{1}$ stable, then, by changing $u(t)$ sufficiently slowly from $u_{1}$ to $u_{2}$ along the curve $\mathcal{U}$, the state $x(t)$ that is close to the point $x^{*}\left(u_{1}\right)$ can be driven into a small neighborhood of $x^{*}\left(u_{2}\right)$. In this paper, it is asserted that, for the same result, slowly varying $u(t)$ is not necessary but it is sufficient that the average of $u(t)$ is

\footnotetext{
1 Local exponential stability is easily relaxed to local asymptotic stability in (Khalil and Kokotović, 1991) once the region of attraction of each $x^{*}(\bar{u})$ does not vanish along the curve $\mathcal{U}$. The latter property has been coined as 'NvBA (Non-vanishing Basin of Attraction) stability' and a verification method of it is proposed in (Shim et $a l ., 2004)$.
} 
slowly varying along the curve $\mathcal{U}$ if the periodic oscillation of $u(t)$ is sufficiently fast.

We note that, in practice, there are several occasions where the slowly-varying-average-input assumption is more suitable than the slowlyvarying-input assumption. A classical example of the slowly-varying-average-input is the PWM (pulse-width modulation) control whose net effect is the average of instantaneous control inputs.

\section{MAIN RESULT}

To effectively describe the system under consideration, we suppose that the system is given by

$$
\begin{gathered}
\dot{x}(t)=f\left(x(t), u_{a}(t), u_{f}\left(\frac{1}{\epsilon} t\right)\right), \\
u_{a} \in \Gamma \subset \mathbb{R}^{m}, u_{f} \in \mathbb{R}^{l},
\end{gathered}
$$

where $f(\cdot, \cdot, \cdot)$ and $u_{a}(\cdot)$ are continuously differentiable, the set $\Gamma$ is connected and compact, and the piecewise continuous function $u_{f}(\cdot)$ is assumed to be periodic with a period $T$ (thus, $u_{f}\left(\frac{1}{\epsilon} t\right)$ is $\epsilon T$-periodic). The small positive parameter $\epsilon$ (to be specified) indicates that the function $u_{f}$ varies fastly. In fact, we are going to rely on threetime scale behavior of the system by specifying $\epsilon$ sufficiently small so that $u_{f}$ oscillates sufficiently fast and by specifying the upper bound of the derivative of $u_{a}$ (i.e., $\left\|\frac{d u_{a}}{d t}\right\| \leq \kappa$ where $\kappa$ is sufficiently small) so that $u_{a}$ varies sufficiently slowly, relatively to the behavior of the system state $x(t)$.

Remark 1. A system having an input $u(t)$ whose average is slowly varying is regarded in this paper as

$$
\dot{x}(t)=f(x(t), u(t))=f\left(x(t), u_{a}(t)+u_{f}\left(\frac{1}{\epsilon} t\right)\right)
$$

where $u_{a}(t)$ is slowly varying and the periodic function $u_{f}(\cdot)$ has zero mean. In this way, this system is cast into (2).

A trick in our development is to regard $\left(\frac{1}{\epsilon} t\right)$ as an independent variable $\tau$ for now. Then, the system (2) is now rewritten as

$$
\dot{x}(t)=f_{a v}\left(x(t), u_{a}(t)\right)+f_{p}\left(x(t), u_{a}(t), u_{f}(\tau)\right)
$$

where $f_{a v}$ is defined as

$$
f_{a v}\left(x, u_{a}\right)=\frac{1}{T} \int_{\sigma}^{\sigma+T} f\left(x, u_{a}, u_{f}(s)\right) d s
$$

with arbitrary $\sigma$, and $f_{p}\left(x, u_{a}, u_{f}\right)=f\left(x, u_{a}, u_{f}\right)-$ $f_{a v}\left(x, u_{a}\right)$. Note that, with this construction,

$$
\int_{\sigma}^{\sigma+T} f_{p}\left(x, u_{a}, u_{f}(s)\right) d s=0
$$

for any $x$ and $u_{a}$.
Now we formally assume the stability property of the averaged system with frozen input $u_{a}$ as follows. Let $B_{r}(x)\left(\bar{B}_{r}(x)\right)$ denote the open (closed) ball centered at $x$ with the radius $r>0$.

Assumption 2. The averaged system

$$
\dot{x}=f_{a v}\left(x, u_{a}\right)
$$

has an isolated equilibrium $x^{*}\left(u_{a}\right)$ for each frozen input $u_{a} \in \Gamma$, that is, $f_{a v}\left(x^{*}\left(u_{a}\right), u_{a}\right)=0$, and there exist a locally Lipschitz function $V: \mathbb{R}^{n} \times$ $\mathbb{R}^{m} \rightarrow \mathbb{R}$ and a positive number $r$ such that, for each $u_{a} \in \Gamma$ and each $x \in B_{r}\left(x^{*}\left(u_{a}\right)\right)$,

$\alpha_{1}\left(\left\|x-x^{*}\left(u_{a}\right)\right\|\right) \leq V\left(x, u_{a}\right) \leq \alpha_{2}\left(\left\|x-x^{*}\left(u_{a}\right)\right\|\right)$

$$
\frac{\partial V}{\partial x} f_{a v}\left(x, u_{a}\right) \leq-\alpha_{3}\left(\left\|x-x^{*}\left(u_{a}\right)\right\|\right), \quad \text { a.e. }
$$

where $\alpha_{i}(\cdot), i=1,2,3$, are class- $\mathcal{K}$ functions.

Theorem 3. Consider the system (2) under Assumption 2 . Given a positive number $\rho$, there exist positive numbers $\epsilon^{*}, \kappa$ and $\delta$ such that, if

$$
\left\|\frac{d u_{a}}{d t}(t)\right\| \leq \kappa
$$

so that the input $u_{a}(t)$ varies sufficiently slowly, and $0<\epsilon<\epsilon^{*}$ so that the input $u_{f}\left(\frac{1}{\epsilon} t\right)$ varies sufficiently fastly, then the state $x(t)$ initiated in a neighborhood of $x^{*}\left(u_{a}(0)\right)$ such that $\left\|x(0)-x^{*}\left(u_{a}(0)\right)\right\| \leq \delta$ stays in a neighborhood of $x^{*}\left(u_{a}(t)\right)$, that is,

$$
\left\|x(t)-x^{*}\left(u_{a}(t)\right)\right\| \leq \rho, \quad{ }^{\forall} t \geq 0 .
$$

PROOF. The proof is taken from (Sanders and Verhulst, 1985) with modification.

First of all, let us define a compact set of our interest,

$$
D:=\left\{\left(x, u_{a}\right) \in \mathbb{R}^{n} \times \Gamma: x \in \bar{B}_{r}\left(x^{*}\left(u_{a}\right)\right)\right\} .
$$

For each element $\left(x, u_{a}\right) \in D$, define $\mu\left(x, u_{a}, \tau\right)$ as

$$
\mu\left(x, u_{a}, \tau\right)=\int_{0}^{\tau} f_{p}\left(x, u_{a}, u_{f}(s)\right) d s,
$$

where $\tau \in \mathbb{R}$. Thus, obviously, it follows that

$$
\begin{aligned}
\frac{\partial \mu}{\partial x}\left(x, u_{a}, \tau\right) & =\int_{0}^{\tau} \frac{\partial f_{p}}{\partial x}\left(x, u_{a}, u_{f}(s)\right) d s, \text { and } \\
\frac{\partial \mu}{\partial u_{a}}\left(x, u_{a}, \tau\right) & =\int_{0}^{\tau} \frac{\partial f_{p}}{\partial u_{a}}\left(x, u_{a}, u_{f}(s)\right) d s .
\end{aligned}
$$

Note that the above integrals are conducted with $x$ and $u_{a}$ fixed. The norms of $\mu\left(x, u_{a}, \tau\right)$, $\frac{\partial \mu}{\partial x}\left(x, u_{a}, \tau\right)$, and $\frac{\partial \mu}{\partial x}\left(x, u_{a}, \tau\right)$ are bounded by some constant $c_{m}>0$ for all $\left(x, u_{a}, \tau\right) \in D \times$ $[0, \infty)$ since $f_{p}\left(x, u_{a}, u_{f}(\tau)\right)$ and its partial derivatives with respect to $x$ and $u_{a}$ are $T$-periodic in $\tau$ and have zero mean.

We consider the change of variables

$$
x=y+\epsilon \mu\left(y, u_{a}, t / \epsilon\right)
$$


where the new variable $y$ is an $n$-dimensional vector. Note that the Jacobian of the map (7) is $\frac{\partial x}{\partial y}\left(y, u_{a}, \tau\right)=I+\epsilon \frac{\partial \mu}{\partial y}\left(y, u_{a}, \tau\right)$. Even though $y$ is defined implicitly, the Jacobian is nonsingular, and the ratio of each leading principal minor of the Jacobian is strictly positive on $D \times[0, \infty)$ for sufficiently small $\epsilon$ since the partial derivative $\frac{\partial \mu}{\partial y}$ is bounded on $D \times[0, \infty)$ as stated earlier. Then, owing to (Kou et al., 1973, Thm. 1), there exists an $\epsilon_{1}>0$ such that, for each $\left(u_{a}, t, \epsilon\right) \in \Gamma \times$ $[0, \infty) \times\left[0, \epsilon_{1}\right]$, the map $y \mapsto x$ is $C^{1}$ and bijective for all $y \in \bar{B}_{r}\left(x^{*}\left(u_{a}\right)\right)$. Differentiating both sides of the equation (7) with respect to time, we have

$$
\begin{aligned}
\dot{x}(t)= & \dot{y}(t)+\epsilon \frac{\partial \mu}{\partial y}\left(y(t), u_{a}(t), t / \epsilon\right) \cdot \dot{y}(t) \\
& +\epsilon \frac{\partial \mu}{\partial u_{a}}\left(y(t), u_{a}(t), t / \epsilon\right) \cdot \dot{u}_{a}(t) \\
& +\epsilon \frac{\partial \mu}{\partial \tau}\left(y(t), u_{a}(t), t / \epsilon\right) \cdot \frac{1}{\epsilon} .
\end{aligned}
$$

We reformulate the above equation as follows

$$
\begin{aligned}
{\left[I+\epsilon \frac{\partial \mu}{\partial y}\left(y(t), u_{a}(t), t / \epsilon\right)\right] \dot{y}(t) } \\
=\dot{x}(t)-\epsilon \frac{\partial \mu}{\partial u_{a}}\left(y(t), u_{a}(t), t / \epsilon\right) \cdot \dot{u}_{a}(t) \\
\quad-\frac{\partial \mu}{\partial \tau}\left(y(t), u_{a}(t), t / \epsilon\right) \\
=f\left(x(t), u_{a}(t), u_{f}(t / \epsilon)\right) \\
\quad-\epsilon \frac{\partial \mu}{\partial u_{a}}\left(y(t), u_{a}(t), t / \epsilon\right) \cdot \dot{u}_{a}(t) \\
\quad-f_{p}\left(y(t), u_{a}(t), u_{f}(t / \epsilon)\right) \\
=f_{a v}\left(y(t), u_{a}(t)\right)-\epsilon \frac{\partial \mu}{\partial u_{a}}\left(y(t), u_{a}(t), t / \epsilon\right) \cdot \dot{u}_{a}(t) \\
\quad+f\left(x(t), u_{a}(t), u_{f}(t / \epsilon)\right)-f\left(y(t), u_{a}(t), u_{f}(t / \epsilon)\right) \\
=f_{a v}\left(y, u_{a}\right)+p_{0}\left(y, u_{a}, t, \epsilon\right) \dot{u}_{a}+p_{1}\left(y, u_{a}, u_{f}, t, \epsilon\right),
\end{aligned}
$$

where $p_{0}\left(y, u_{a}, t, \epsilon\right)=-\epsilon \frac{\partial \mu}{\partial u_{a}}$ and $p_{1}\left(y, u_{a}, u_{f}, t, \epsilon\right)=$ $\left[f\left(y+\epsilon \mu, u_{a}, u_{f}\right)-f\left(y, u_{a}, u_{f}\right)\right]$. The function $p_{1}$ is written as

$$
p_{1}\left(y, u_{a}, u_{f}, t, \epsilon\right)=\bar{f}\left(y, u_{a}, u_{f}, \epsilon \mu\right) \cdot \epsilon \mu,
$$

where $\bar{f}$ is a matrix of continuous functions whose existence follows from the continuous differentialibity of $f$. It can be seen easily that

$$
\begin{aligned}
\left\|p_{0}\left(y, u_{a}, t, \epsilon\right)\right\| & \leq \epsilon c_{m} \\
\left\|p_{1}\left(y, u_{a}, u_{f}, t, \epsilon\right)\right\| & \leq \epsilon c_{f} c_{m},
\end{aligned}
$$

for all $\left(y, u_{a}\right) \in D, t \in[0, \infty)$ and $\epsilon \in\left[0, \epsilon_{1}\right]$, where

$$
c_{f}=\sup _{\left(y, u_{a}\right) \in D, 0 \leq \tau, 0 \leq \epsilon \mu \leq \epsilon_{1} c_{m}}\left\|\bar{f}\left(y, u_{a}, u_{f}(\tau), \epsilon \mu\right)\right\|
$$

$\left(c_{f}<\infty\right.$ since $\bar{f}$ is continuous and $u_{f}$ is piecewise continuous).

We use the function $V$ in Assumption 2 to analyze the transformed system. The time-derivative of $V$ along the system in $y$-coordinates is given by

$$
\begin{aligned}
\dot{V}\left(y, u_{a}\right)=\frac{\partial V}{\partial y} \dot{y}(t)+\frac{\partial V}{\partial u_{a}} \dot{u}_{a}(t) \\
=\frac{\partial V}{\partial y}\left[I+\epsilon \frac{\partial \mu}{\partial y}\right]^{-1}\left(f_{a v}+p_{0} \dot{u}_{a}+p_{1}\right)+\frac{\partial V}{\partial u_{a}} \dot{u}_{a}(t) \\
=\frac{\partial V}{\partial y} f_{a v}+\frac{\partial V}{\partial y}\left(\left[I+\epsilon \frac{\partial \mu}{\partial y}\right]^{-1}-I\right) f_{a v} \\
\quad+\frac{\partial V}{\partial y}\left[I+\epsilon \frac{\partial \mu}{\partial y}\right]^{-1}\left(p_{0} \dot{u}_{a}+p_{1}\right)+\frac{\partial V}{\partial u_{a}} \dot{u}_{a}(t) .
\end{aligned}
$$

Since the function $V$ is locally Lipschitz, its partial derivatives $\frac{\partial V}{\partial y}$ and $\frac{\partial V}{\partial u_{a}}$ are bounded on $D$ almost everywhere, and we let

$$
\begin{aligned}
c_{v} & =\operatorname{ess} \cdot \sup _{\left(y, u_{a}\right) \in D}\left\|\frac{\partial V}{\partial y}\left(y, u_{a}\right)\right\|, \\
c_{w} & =\operatorname{ess} \cdot \sup _{\left(y, u_{a}\right) \in D}\left\|\frac{\partial V}{\partial u_{a}}\left(y, u_{a}\right)\right\|, \\
c_{a} & =\operatorname{ess} \cdot \sup _{\left(y, u_{a}\right) \in D}\left\|f_{a v}\left(y, u_{a}\right)\right\| .
\end{aligned}
$$

In addition, for $\epsilon<\frac{1}{c_{m}}$, we have

$$
\begin{aligned}
& \left\|\left[I+\epsilon \frac{\partial \mu}{\partial y}\right]^{-1}\right\| \leq \frac{1}{1-\epsilon c_{m}}, \text { and } \\
& \left\|\left[I+\epsilon \frac{\partial \mu}{\partial y}\right]^{-1}-I\right\| \leq \frac{\epsilon c_{m}}{1-\epsilon c_{m}} .
\end{aligned}
$$

Consequently, from Assumption 2, (8) and (9), it follows that

$$
\begin{aligned}
\dot{V}\left(y(t), u_{a}(t)\right) \leq & -\alpha_{3}\left(\left\|y(t)-x^{*}\left(u_{a}\right)\right\|\right) \\
& +c_{v} \frac{\epsilon c_{m}}{1-\epsilon c_{m}} c_{a}+c_{v} \frac{1}{1-\epsilon c_{m}} \epsilon c_{m}\left\|\dot{u}_{a}\right\| \\
& +c_{v} \frac{1}{1-\epsilon c_{m}} \epsilon c_{f} c_{m}+c_{w}\left\|\dot{u}_{a}\right\|,
\end{aligned}
$$

on $D$, almost everywhere.

Now, without loss of generality, suppose that the given number $\rho \leq r$. We can take a pair of positive numbers $\left(\epsilon_{2}, \kappa\right)$ such that

$\frac{\epsilon_{2} c_{m}}{1-\epsilon_{2} c_{m}} c_{v}\left(c_{a}+\kappa+c_{f}\right)+c_{w} \kappa \leq\left(\alpha_{3} \circ \alpha_{2}^{-1} \circ \alpha_{1}\right)\left(\frac{\rho}{2}\right)$.

Then,

$\dot{V}\left(y(t), u_{a}(t)\right) \leq-\alpha_{3}\left(\alpha_{2}^{-1}(V)\right)+\left(\alpha_{3} \circ \alpha_{2}^{-1} \circ \alpha_{1}\right)\left(\frac{\rho}{2}\right)$,

which implies that, if $V\left(y(0), u_{a}(0)\right) \leq \alpha_{1}(\rho / 2)$, then $V\left(y(t), u_{a}(t)\right) \leq \alpha_{1}(\rho / 2)$ for all $t \geq 0$. Therefore, letting $\delta=\left(\alpha_{2}^{-1} \circ \alpha_{1}\right)\left(\frac{\rho}{2}\right)$, we have

$$
\left\|y(t)-x^{*}\left(u_{a}(t)\right)\right\| \leq \frac{\rho}{2},
$$

for all $t \geq 0$ if $\left\|y(0)-x^{*}\left(u_{a}(0)\right)\right\| \leq \delta$ and $\epsilon<\min \left\{\epsilon_{1}, \epsilon_{2}, \frac{1}{c_{m}}\right\}$.

To complete the proof, the analysis is given in $x$ coordinate. From $(7), x(0)=y(0)$. Thus, if $\| x(0)-$ $x^{*}\left(u_{a}(0)\right) \| \leq \delta$, then $\left\|y(t)-x^{*}\left(u_{a}(t)\right)\right\| \leq \frac{\rho}{2}$ and $\left(y(t), u_{a}(t)\right) \in D$ for all $t \geq 0$, which implies that 


$$
\begin{aligned}
\left\|x(t)-x^{*}\left(u_{a}(t)\right)\right\| & \leq\|x(t)-y(t)\|+\left\|y(t)-x^{*}\left(u_{a}\right)\right\| \\
& \leq\left\|\epsilon \mu\left(y(t), u_{a}(t), t / \epsilon\right)\right\|+\frac{\rho}{2} \\
& \leq \epsilon c_{m}+\frac{\rho}{2} .
\end{aligned}
$$

Therefore, we finally set

$$
\epsilon^{*}=\min \left\{\epsilon_{1}, \epsilon_{2}, \frac{1}{c_{m}}, \frac{\rho}{2 c_{m}}\right\},
$$

and we have

$$
\left\|x(t)-x^{*}\left(u_{a}(t)\right)\right\| \leq \rho,
$$

for all $t \geq 0$ provided that $\epsilon<\epsilon^{*}$.

\section{CONCLUSION}

In this note, we use the features of the slowlyvarying periodic input and the averaging theory to prove that if each equilibrium point for the frozen system of our concern is asymptotically stable, its stability property is not severely disturbed by inputs having slowly-varying average. Finally, it seems that the result in this note can be extended using 'general' averaging theory applicable to the case that $u_{f}(t)$ may not be periodic.

\section{REFERENCES}

Kelemen, M. (1986). A stability property. IEEE Trans. Automat. Contr. 31, 766-768.

Khalil, H. (1996). Nonlinear Systems. 2nd ed.. Prentice-Hall.

Khalil, H. and P. Kokotović (1991). On stability properties of nonlinear systems with slowly varying inputs. IEEE Trans. Automat. Contr. 36, 229.

Kou, S., D. Elliott and T. Tarn (1973). Observability of nonlinear systems. Information and Control 22, 89-99.

Lawrence, D. and W. Rugh (1990). On a stability theorem of nonlinear systems with slowly varying inputs. IEEE Trans. Automat. Contr. 7, 860-864.

Sanders, J. and F. Verhulst (1985). Averaging methods in nonlinear dynamical systems. Springer-Verlag.

Shim, H., H. Chang and J. Seo (2004). Nonvanishing basin of attraction with respect to a parametric variation and center manifold. IEEE Conf. on Decision and Control, $p p$. 1048-1054. 